\title{
Achtung Falle: Soll ich Sie krank schreiben?
}

\section{Toni Nadig}

Dr. rer. soc., Arbeits- und Betriebspsychologie mit Promotion in klinischer Neuropsychologie; Fachpsychologe in Laufbahn- und Personalpsychologie FSP

\begin{abstract}
Der Verlust des Arbeitsplatzes ist für Betroffene häufig eine Lebenskrise, nicht selten begleitet durch gesundheitliche Beeinträchtigungen. Eine Krankschreibung scheint da angebracht. Entweder fragt der Betroffene den Arzt direkt - oder der Mediziner bietet es von sich aus an. Doch das kann auch ein Fehler sein, der zusätzliche Hürden schafft.
\end{abstract}

Die Arbeitswelt hat sich in den letzten Jahren fundamental verändert. Die Globalisierung führt zur Verlagerung von Jobs ins Ausland, der Strukturwandel verändert die Anforderungsprofile. Auch die Wertewelt hat sich verändert: Loyalität und Stabilität stehen weniger im Vordergrund, gefragt sind Veränderungsbereitschaft und Flexibilität. Für Arbeitnehmer eine grosse Herausforderung, die manche auch überfordert.

\section{Etwa ein Drittel der Stellensuchenden leidet unter psychosomatischen Symptomen.}

Auf der Seite der Arbeitgeber sind Entlassungen zur Normalität geworden. Der Arbeitnehmer wird zunehmend zur Ressource, die flexibel beschafft werden

\section{Dois-je vous mettre en arrêt maladie?}

La perte d'un emploi est pour certains parfois si traumatisante qu'ils décident de consulter un médecin pour cause de symptômes somatiques et psychosomatiques et se font mettre en arrêt maladie. II advient que des médecins leur proposent un arrêt maladie afin de leur donner plus de temps pour la recherche d'un nouvel emploi. Un tel congé de maladie apparemment bénéfique peut avoir des conséquences négatives importantes. De fait, la personne en congé de maladie durant plus de trois semaines doit le déclarer auprès de la nouvelle institution de prévoyance professionnelle et risque alors de se voir attribuer une réserve allant jusqu'à cinq ans pour cause de maladie psychique. II est plus utile de conseiller aux personnes concernées de consulter un service d'orientation professionnelle ou de leur recommander un replacement externe. Si les problèmes de santé sont susceptibles de provoquer une incapacité de travail de longue durée ou de rendre la recherche d'un emploi difficile, il est possible de demander à l'Al une consultation à bas seuil dans le cadre d'une détection et d'une intervention précoces. muss. Arbeitslosigkeit kann heute jeden und jede treffen. Die entstehende Situation ist für die Betroffenen schwierig: die Angst vor dauerhafter Arbeitslosigkeit, die Frage, ob eine neue und auch angemessene Stelle zu finden ist, die Sorgen, ob man seinen Lebensunterhalt weiter finanzieren kann. Versagensszenarien spielen eine Rolle, nicht selten kommt Scham vor der Familie und den Freunden hinzu. Der Verlust des Arbeitsplatzes kann zu einer Lebenskrise führen, die durchaus mit dem Verlust eines nahen Angehörigen oder dem Scheitern einer Ehe vergleichbar ist. Etwa ein Drittel der Stellensuchenden leidet unter psychosomatischen Symptomen. Es ist nicht verwunderlich, wenn Betroffene in einer solchen Situation auf Anregung ihres Hausarztes (oder auch auf eigenen Wunsch) für Wochen oder gar Monate krankgeschrieben werden. Einerseits brauchen sie Entlastung und Erholung, andererseits gewinnen sie Zeit für die Stellensuche. Diese Art des Vorgehens scheint auf den ersten Blick gut und vernünftig zu sein. Und doch ist es oft der falsche Weg. Natürlich gibt es Menschen, die ernsthafte physische oder psychische Erkrankungen haben, die eine Krankschreibung zwingend erfordern. Fakt ist aber auch: Verfügt der Betroffene über auf dem Arbeitsmarkt gefragte Kompetenzen, kann er meistens wieder recht schnell eine zu seinen Neigungen passende Stelle finden. Lassen sich Stellensuchende bei der Neuorientierung richtig beraten, schaffen es rund 90 Prozent von ihnen innerhalb eines Jahres zurück in den Job. Wer sich hingegen länger als drei Wochen krankschreiben lässt, schafft eine Reihe von Problemen, die ihm oft gar nicht bewusst sind.

Zunächst ist es als "Kranker» schwierig, eine neue Stelle zu finden. Welcher Arbeitgeber möchte eine Person einstellen, von der er annehmen muss, dass sie in 


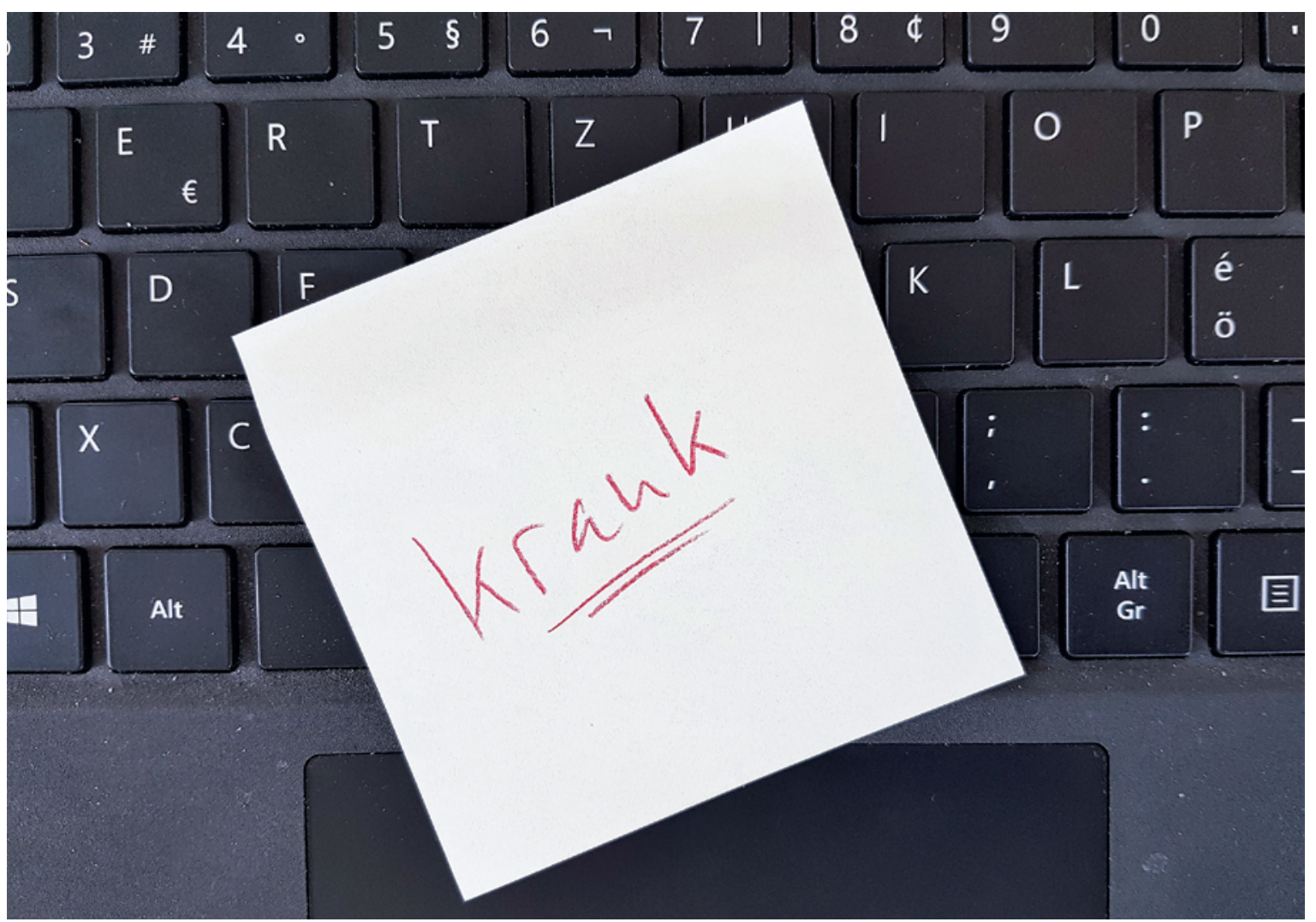

Zukunft regelmässig ausfällt? Besonders problematisch ist das bei Führungskräften. Hier ist die physische und psychische Belastbarkeit quasi schon Bestandteil des Arbeitsvertrages. Spricht sich eine Krankschreibung einer solchen Person herum, sinken die Chancen auf einen adäquaten Job gegen Null. Das ist sogar verständlich, wenn man berücksichtigt, dass beispielsweise die finanziellen Konsequenzen eines Burnout-Falls den Arbeitgeber im Schnitt ein ganzes Jahresgehalt kosten können.

Ein weiteres Problem: Man bekommt Schwierigkeiten

\section{Lassen sich Stellensuchende richtig beraten, schaffen es rund 90 Prozent von ihnen innerhalb eines Jahres zurück in den Job.}

mit der Vorsorgeeinrichtung (2. Säule): Wer eine neue Stelle antritt, wechselt üblicherweise die Pensionskasse. Beim Eintritt in eine neue Pensionskasse muss eine Gesundheitserklärung abgegeben werden. So wird gefragt, ob man aktuell hundertprozentig arbeitsfähig ist und in den letzten zwölf Monaten wegen einer Krankheit mehr als drei Wochen teilweise oder vollständig arbeitsunfähig war. Ausserdem wollen die Vorsorgeeinrichtungen wissen, unter welchen Krankheiten man in den letzten drei Jahren gelitten hat. Die
Pensionskassen haben das Recht, eine Gesundheitsprüfung durchführen zu lassen. War der Betroffene im abgefragten Zeitraum beispielsweise wegen eines Burnouts oder einer Depression krankgeschrieben, kann die Pensionskasse die Risikoleistungen deutlich reduzieren oder sogar einen fünfjährigen Vorbehalt bezüglich der entsprechenden Leiden erklären. Wer bei der Gesundheitsprüfung falsche Angaben macht, riskiert die komplette Streichung möglicher Leistungen.

Das hat Konsequenzen, schaut man sich das Prinzip der Invalidenversicherung in der Schweiz an. Die Invalidenversicherung kennt mit der Früherfassung und Frühintervention ein präventives Instrument, um Personen mit ersten Anzeichen einer möglichen längeren Krankheit oder drohenden Invalidität oder voraussichtlicher Arbeitslosigkeit infolge einer Erkrankung rasch zu erfassen. Ziel ist es, den betroffenen Personen mit Hilfe von geeigneten Massnahmen einen Verbleib im Arbeitsprozess oder eine rasche Wiedereingliederung zu ermöglichen. Der behandelnde Arzt kann Massnahmen einleiten, wenn sein Patient wegen des Erhalts des Arbeitsplatzes oder der Suche nach einem neuen Arbeitsplatz Beratung braucht.

Stellt sich heraus, dass an einer Invalidisierung kein Weg vorbeiführt, kommt es zu einer Berentung. Die bekommt man frühestens nach einem Jahr, meistens 
dauert der Prozess deutlich länger. Die Sozialversicherung hat vor allem das Ziel, die Menschen zurück ins Arbeitsleben zu begleiten. Nur, wenn das nicht möglich ist, stellt sich die Frage einer Invalidenrente.

Diese Rente reicht allerdings kaum aus, um den Lebens-

\section{Tatsächlich brauchen einige Betroffene psychotherapeutische oder sogar pharmakologische Unterstützung - das geht aber durchaus ohne Krankschreibung.}

unterhalt zu bestreiten. Normalerweise wird die Rente durch die Pensionskasse, in die mehr als zwei Drittel aller Schweizer einzahlen, ergänzt - allerdings nicht, wenn ein wirksamer Vorbehalt existiert. Betroffene können somit rasch zu Sozialfällen werden - und das nur wegen einiger Wochen vermeidbarer Krankschreibung.

Meine Gespräche mit Hausärzten ergaben, dass ihnen die geschilderte Problematik oft nicht bewusst ist und sie damit gar nicht wissen, was sie mit der an sich gutgemeinten Entlastung durch Krankschreibung anrichten. Bespricht man das Thema mit betroffenen Stellensuchenden, dann ist das «Kranksein» meistens keine Option mehr. Tatsächlich brauchen einige Betroffene psychotherapeutische oder sogar pharmakologische Unterstützung - das geht aber durchaus ohne Krankschreibung.

Man sollte sich vor Augen halten, dass in der Schweiz täglich etwa hundert Stellen verlorengehen, aber gleichzeitig etwa hundertfünfzig neue entstehen. Das Problem ist: Die Anforderungsprofile der neuen Stellen unterscheiden sich häufig von denen, die verloren gehen. Was müssen Arbeitssuchende heute also tun? Die Stellensuche sollte sofort nach dem Stellenverlust beginnen. Allerdings sollte man, bevor man aktiv auf dem Arbeitsmarkt sucht, folgende Fragen beantworten: Was kann ich? Was will ich? Was passt zu mir? Wo kann ich meine Kompetenzen erfolgreich anbieten und einbringen? Diese vierte Frage wird bisweilen ganz vergessen oder vernachlässigt. So einfach sich diese Fragen aufschreiben und lesen lassen - vielen Arbeitssuchenden gelingt es nicht, ohne Hilfe die passenden Antworten zu finden. Die richtigen Ansprechpartner sind hier je nach Qualifikation des Betroffenen vor allem Regionale Arbeitsvermittlungszentren (RAV), Berufsberater oder Outplacement-Experten und natürlich die Sozialversicherung mit der Früherfassung - die bei der Standortbestimmung, Neuorientierung und Stellensuche helfen.

Fazit: Hausärzte müssen so informiert sein, dass sie hilfesuchende Personen motivieren und dabei unterstützen, sofort mit einer Neuorientierung zu beginnen. Die Unterstützung kann in Form einer Laufbahn-

Man sollte sich vor Augen halten, dass in der Schweiz täglich etwa hundert Stellen verlorengehen, aber gleichzeitig etwa hundertfünfzig neue entstehen.

beratung oder eines Outplacements erfolgen und gegebenenfalls mit Psychotherapie und Medikamenten begleitend unterstützt werden. Die Krankschreibung sollte die Ausnahme und letzte Massnahme sein. Durch rasches Handeln kann unter Umständen einer Verschlechterung des gesundheitlichen Zustandes entgegengewirkt und ein Ausscheiden aus der Arbeitswelt verhindert werden.

Disclosure statement

Toni Nadig ist Gründer und Partner der Outplacementfirma Dr. Nadig \& Partner AG in Zürich

\section{Bildnachweis}

Foto: Anna Sax 